Vol. 96 (1984), 167-174

\title{
A ZETA FUNCTION CONNECTED WITH THE EIGENVALUES OF THE LAPLACE-BELTRAMI OPERATOR ON THE FUNDAMENTAL DOMAIN OF THE MODULAR GROUP
}

\author{
AKIO FUJII
}

\section{§1. Introduction}

Let $\lambda_{0}=0 \leqq \lambda_{1} \leqq \lambda_{2} \leqq \cdots$ run over the eigenvalues of the discrete spectrum of the Laplace-Beltrami operator on $L^{2}(H / \Gamma)$, where $H$ is the upper half of the complex plane and we take $\Gamma=P S L(2, Z)$. It is well known that $\lambda_{1}>\frac{1}{4}$. We put $\lambda_{j}=\frac{1}{4}+r_{j}^{2}$ for $j \geqq 0$. Let $\alpha$ be a positive number. Here we are concerned with the zeta function defined by

$$
Z_{\alpha}(s)=\sum_{r_{j}>0} \frac{\sin \left(\alpha r_{j}\right)}{r_{j}^{s}}
$$

We shall prove the following theorem.

TheOREM. For any positive $\alpha, Z_{\alpha}(s)$ is entire.

This should be compared with Minakshisundaram and Pleijel [8], Guinand [4] and Delsarte [1] and also with the author's result which states that on the Riemann Hypothesis $\sum_{r>0}\left(\sin (\alpha \gamma) / \gamma^{s}\right)$ is entire for any positive $\alpha$, where $\gamma$ runs over the imaginary parts of the zeros of the Riemann zeta function $\zeta(s)$ (cf. [3]).

We recall first Selberg's trace formula. Let $h(r)$ satisfy the conditions;

1) $h(r)=h(-r)$

2) $h(r)$ is analytic in the strip $|\operatorname{Im} r|<\frac{1}{2}+\varepsilon, \varepsilon>0$

3) $h(r)=O\left(\left(1+|r|^{2}\right)^{-1-\varepsilon}\right)$ in this strip.

Then we have

$$
\begin{aligned}
\sum h\left(r_{j}\right)= & \frac{1}{6} \int_{-\infty}^{\infty} r \text { th }(\pi r) h(r) d r \\
& +\int_{-\infty}^{\infty}\left(\frac{1}{2}+\frac{2}{3 \sqrt{3}}\left(e^{\pi / / 3}+e^{-\pi r / 3}\right)\right) \frac{h(r)}{e^{\pi r}+e^{-\pi r}} d r
\end{aligned}
$$

Received December 8, 1983. 


$$
\begin{aligned}
& +2 \sum_{\left\{P_{0}\right\}} \sum_{k=1}^{\infty} \frac{\log N\left(P_{0}\right)}{N\left(P_{0}\right)^{k / 2}-N\left(P_{0}\right)^{-k / 2}} g\left(k \log N\left(P_{0}\right)\right) \\
& +\frac{1}{2 \pi} \int_{-\infty}^{\infty} h(r) \frac{\varphi^{\prime}}{\varphi}\left(\frac{1}{2}+i r\right) d r-\frac{1}{\pi} \int_{-\infty}^{\infty} h(r) \frac{\Gamma^{\prime}}{\Gamma}(1+i r) d r \\
& -2 \log 2 g(0)+\frac{1}{2}\left(1-\varphi\left(\frac{1}{2}\right)\right) h(0),
\end{aligned}
$$

where the left hand side is over all the solutions $r_{j}$ of all the equations $\lambda_{j}=\frac{1}{4}+r_{j}^{2},\left\{P_{0}\right\}$ runs over all primitive hyperbolic conjugacy classes in $\Gamma, N\left(P_{0}\right)$ is the square of the eigenvalue (greater than one) of a representative element $P_{0}$,

$$
g(u)=\frac{1}{2 \pi} \int_{-\infty}^{\infty} e^{-i r u} h(r) d r, \quad \varphi(s)=\sqrt{\pi} \frac{\Gamma\left(s-\frac{1}{2}\right) \zeta(2 s-1)}{\Gamma(s) \zeta(2 s)}
$$

(cf. Selberg [9] and Hejhal [6]). Let $Z(s)$ be the Selbarg's zeta function defined by

$$
Z(s)=\prod_{\left\{P_{0}\right\}} \prod_{k=0}^{\infty}\left(1-N\left(P_{0}\right)^{-k-s}\right) \quad \text { for } \operatorname{Re} s>1 .
$$

Then Selberg's trace formula describes the location of the poles and the zeros of $Z(s)$ and gives the functional equation of $Z(s)$. Using these, one can deduce the following formula for $T>0$,

$$
\begin{aligned}
N(T) \equiv & |\{0 \leqq r \leqq T\}| \\
= & \frac{1}{4 \pi} \int_{0}^{T}\left(\frac{\varphi^{\prime}}{\varphi}\left(\frac{1}{2}+i r\right)+\frac{\varphi^{\prime}}{\varphi}\left(\frac{1}{2}-i r\right)\right) d r \\
& +S_{z}(T)+\frac{1}{12} T^{2}-\frac{1}{\pi} T \log T+\frac{\log (e / 2)}{\pi} T+w(T),
\end{aligned}
$$

where $r$ runs over $r_{j}, S_{Z}(T)=(1 / \pi) \arg Z\left(\frac{1}{2}+i T\right)$ as usual and $w(T)$ satisfies $w^{\prime}(T) \ll T^{-2}$ for $T>T_{0}$ (cf. Venkov [11]). Using this formula, one gets the following result which can be proved by the same method as the author's [2];

$$
\begin{aligned}
\sum_{0<r \leqq T} e^{i \alpha r}= & \frac{1}{\pi} \frac{\Lambda\left(e^{\alpha / 2}\right)}{e^{\alpha / 2}} T+\frac{e^{i \alpha T}}{6 i \alpha} T \\
& +\frac{1}{2 \pi} e^{-\alpha / 2}\left(\sum_{\{P\}, N(P)=e^{\alpha}} \tilde{\Lambda}(P)\right) T+O(T / \log T)
\end{aligned}
$$

where $T>T_{0}, \Lambda(x)$ is the von Mangoldt function, $\{P\}$ runs over all hyperbolic conjugacy classes and we put 


$$
\Lambda(P)=\frac{\log N\left(P_{0}\right)}{1-N\left(P_{0}\right)^{-k}} \quad \text { and } \quad N(P)=N\left(P_{0}\right)^{k} \quad \text { if } P=P_{0}^{k}
$$

with an integer $k \geqq 1$. By this we see that $Z_{\alpha}(s)$ is regular in $\operatorname{Re} s>1$. We shall prove its analytic continuation using the Selberg's trace formula.

Finally, we remark that $Z_{\alpha}(1)$ or $Z_{\alpha}(0)$ can be evaluated as a biproduct of the proof of the above theorem and they have some significant arithmetic meanings. ${ }^{(*)}$

\section{§2. Proof of Theorem}

We use Selberg's trace formula with

$$
h(r)=e^{-\left(1 / 4+r^{2}\right) x} \sin (\alpha r) r .
$$

Then

$$
\begin{aligned}
g(u)= & -\frac{(u-\alpha)}{8 \sqrt{\pi} x^{3 / 2}} e^{-(u-\alpha)^{2} / 4 x} e^{-(1 / 4) x} \\
& +\frac{(u+\alpha)}{8 \sqrt{\pi} x^{3 / 2}} e^{-(u+\alpha)^{2} / 4 x} e^{-(1 / 4) x}
\end{aligned}
$$

and

$$
\begin{aligned}
& 2 \sum_{r>0} e^{-\left(1 / 4+r^{2}\right) x} \sin (\alpha r) r \\
&= \frac{1}{2}\left(e^{\alpha / 2}-e^{-\alpha / 2}\right)+\frac{1}{6} \int_{-\infty}^{\infty} r \operatorname{th}(\pi r) e^{-\left(1 / 4+r^{2}\right) x} \sin (\alpha r) r d r \\
&+\int_{-\infty}^{\infty}\left(\frac{1}{2}+\frac{2}{3 \sqrt{3}}\left(e^{(1 / 3) r}+e^{-(1 / 3) r}\right)\right) \frac{e^{-\left(1 / 4+r^{2}\right) x} \sin (\alpha r) r}{e^{\pi r}+e^{-\pi r}} d r \\
&+\frac{x^{-3 / 2}}{4 \sqrt{\pi}} \sum_{\{P\}} \frac{\tilde{\Lambda}(P)}{\sqrt{N(P)}}(\alpha-\log N(P)) e^{-(\alpha-\log N(P)) 2 / 4 x} e^{-(1 / 4) x} \\
&+\frac{x^{-3 / 2}}{4 \sqrt{\pi}} \sum_{\{P\}} \frac{\tilde{\Lambda}(P)}{\sqrt{N(P)}}(\alpha+\log N(P)) e^{-(\alpha+\log N(P)) 2 / 4 x} e^{-(1 / 4) x} \\
&+\frac{\log \pi / 2}{2 \sqrt{\pi}} \alpha x^{-3 / 2} e^{-\alpha^{2} / 4 x} e^{-(1 / 4) x} \\
&-\frac{1}{\pi} \int_{-\infty}^{\infty} e^{-\left(1 / 4+r^{2}\right) x} \sin (\alpha r) r\left(\frac{\Gamma^{\prime}}{\Gamma}\left(\frac{1}{2}+i r\right)+\frac{\Gamma^{\prime}}{\Gamma}(1+i r)\right) d r \\
&+\frac{1}{2 \sqrt{\pi}} x^{-3 / 2} \sum_{n=2}^{\infty} \frac{\Lambda(n)}{n}(\alpha+2 \log n) e^{-(2 \log n+\alpha)^{2} / 4 x} e^{-(1 / 4) x} \\
&+\frac{1}{2 \sqrt{\pi}} x^{-3 / 2} \sum_{n=2}^{\infty} \frac{\Lambda(n)}{n}(\alpha-2 \log n) e^{-(2 \log n-\alpha)^{2} / 4 x} e^{-(1 / 4) x} .
\end{aligned}
$$

(*) The results are announced in the author's "Zeros, Eigenvalues and Arithmetic" (Proc. of Japan Academy, 60 Ser. A (1984) p. 22-25). 
Now we consider the integral

$$
I \equiv \int_{0}^{\infty} x^{s-1} \sum_{r>0} e^{-r^{2} x} \sin (\alpha r) r d x .
$$

We remark that

$$
\begin{aligned}
\sum_{r>0} r \int_{0}^{\infty} x^{\sigma-1} e^{-r^{2} x} d x & =\sum_{r>0} \frac{1}{r^{2 \sigma-1}} \int_{0}^{\infty} x^{\sigma-1} e^{-x} d x \\
& =\Gamma(\sigma) \sum_{r>0} \frac{1}{r^{2 \sigma-1}} \quad \text { for } \sigma>3 / 2 .
\end{aligned}
$$

Hence for $\operatorname{Re} s>3 / 2$,

$$
I=Z_{\alpha}(2 s-1) \Gamma(s) .
$$

Now

$$
\begin{aligned}
I & =\left(\int_{0}^{1}+\int_{1}^{\infty}\right) x^{s-1}\left(\sum_{r>0} e^{-r^{2} x} \sin (\alpha r) r\right) d x \\
& =I_{1}+I_{2}, \text { say } .
\end{aligned}
$$

$I_{2}$ is entire.

$$
\begin{aligned}
I_{1}= & \frac{1}{4}\left(e^{\alpha / 2}-e^{-\alpha / 2}\right) \int_{0}^{1} x^{s-1} e^{(1 / 4) x} d x \\
& +\frac{1}{12} \int_{0}^{1} x^{s-1} \int_{-\infty}^{\infty} r \operatorname{th}(\pi r) e^{-r^{2} x} \sin (\alpha r) r d r d x \\
& +\int_{0}^{1} x^{s-1} \int_{-\infty}^{\infty}\left(\frac{1}{4}+\frac{1}{3 \sqrt{3}}\left(e^{\pi r / 3}+e^{-\pi r / 3}\right)\right) \frac{e^{-r^{2} x} \sin (\alpha r) r}{e^{\pi r}+e^{-\pi r}} d r d x \\
& +\frac{1}{8 \sqrt{\pi}} \int_{0}^{1} x^{s-5 / 2} \sum_{\{P\}} \frac{\tilde{\Lambda}(P)}{\sqrt{N(P)}}(\alpha-\log N(P)) e^{-(\alpha-\log N(P))^{2 / 4 x}} d x \\
& +\frac{1}{8 \sqrt{\pi}} \int_{0}^{1} x^{s-5 / 2} \sum_{\{P\}} \frac{\tilde{\Lambda}(P)}{\sqrt{N(P)}}(\alpha+\log N(P)) e^{-(\alpha+\log N(P))^{2 / 4 x}} d x \\
& +\frac{\log (\pi / 2)}{4 \sqrt{\pi}} \alpha \int_{0}^{1} x^{s-5 / 2} e^{-\alpha^{2} / 4 x} d x \\
& -\frac{1}{2 \pi} \int_{0}^{1} x^{s-1} \int_{-\infty}^{\infty} e^{-r^{2} x} \sin (\alpha r) r\left(\frac{\Gamma^{\prime}}{\Gamma}\left(\frac{1}{2}+i r\right)+\frac{\Gamma^{\prime}}{\Gamma}(1+i r)\right) d r d x \\
& +\frac{1}{4 \sqrt{\pi}} \int_{0}^{1} x^{s-5 / 2} \sum_{n=2}^{\infty} \frac{\Lambda(n)}{n}\left((\alpha-2 \log n) e^{-(\alpha-2 \log n)^{2 / 4 x}}\right. \\
= & I_{3}+I_{4}+\cdots+I_{9}+\left(I_{10}+I_{11}\right), \quad \text { say } .
\end{aligned}
$$


We see at first that $I_{3} / \Gamma(s)$ and $I_{5} / \Gamma(s)$ are entire. Since

$$
I_{6}=\frac{1}{8 \sqrt{\pi}} \int_{1}^{\infty} x^{-s+1 / 2} \sum_{\{P\}} \frac{\tilde{\Lambda}(P)}{\sqrt{N(P)}}(\alpha-\log N(P)) e^{-(\log N(P)-\alpha)^{2} x / 4} d x
$$

$I_{6}$ is entire. Similarly, $I_{7}, I_{8}, I_{10}$ and $I_{11}$ are entire.

We shall treat $I_{9}$ next.

$$
\begin{aligned}
I_{9}= & -\frac{1}{2 \pi} \int_{0}^{1} x^{s-1}\left(\int_{0}^{1}+\int_{1}^{\infty}\right) e^{-r^{2 x}} \sin (\alpha r) r \\
& \cdot\left(\frac{\Gamma^{\prime}}{\Gamma}\left(\frac{1}{2}+i r\right)+\frac{\Gamma^{\prime}}{\Gamma}\left(\frac{1}{2}-i r\right)+\frac{\Gamma^{\prime}}{\Gamma}(1+i r)+\frac{\Gamma^{\prime}}{\Gamma}(1-i r)\right) d r d x \\
= & I_{12}+I_{13}, \quad \text { say. }
\end{aligned}
$$

$I_{12} / \Gamma(s)$ is entire.

By Stirling's formula, we see that

$$
\begin{aligned}
I_{13} & =-\frac{1}{2 \pi} \int_{0}^{1} x^{s-1} \int_{1}^{\infty} e^{-r^{2 x}} \sin (\alpha r) r\left(4 \log r+\sum_{k=1}^{\infty} \frac{b_{k}}{r^{2 k}}\right) d r d x \\
& =I_{14}(s)+I_{15}(s), \quad \text { say. }
\end{aligned}
$$

We remark that $I_{15}(s) / \Gamma(s)$ is entire.

$$
\begin{aligned}
I_{14}(s) & =-\frac{1}{\pi} \int_{0}^{1} x^{s-2}\left(\nu_{1}(x)+\alpha \nu_{2}(x)-\alpha \nu_{3}(x)\right) d x \\
& =-\frac{1}{\pi} J_{1}(s)-\frac{\alpha}{\pi} J_{2}(s)+\frac{\alpha}{\pi} J_{3}(s), \quad \text { say },
\end{aligned}
$$

where we put

$$
\begin{aligned}
& \nu_{1}(x)=\int_{1}^{\infty} e^{-r^{2} x} \sin (\alpha r) r^{-1} d r \\
& \nu_{2}(x)=\int_{0}^{\infty} e^{-r^{2} x} \cos (\alpha r) \log r d r
\end{aligned}
$$

and

$$
\nu_{3}(x)=\int_{0}^{1} e^{-r^{2} x} \cos (\alpha r) \log r d r .
$$

$J_{1}(s) / \Gamma(s)$ is entire except a simple pole at $s=1$. Since

$$
J_{3}(s)=\frac{1}{s-1} \nu_{3}(1)-\frac{1}{s-1} \int_{0}^{1} x^{s-1} \nu_{3}^{\prime}(x) d x
$$

for $\operatorname{Re} s>1, J_{3}(s) / \Gamma(s)$ is entire except a simple pole at $s=1$. 


$$
\begin{aligned}
J_{2}(s)= & -\frac{\sqrt{\pi}}{4} \int_{0}^{1} x^{s-5 / 2} \log x e^{-\alpha^{2} / 4 x} d x \\
& +\frac{\sqrt{\pi}}{4} \int_{1}^{\infty} x^{-s+1 / 2}\left(\int_{0}^{1} e^{-y} e^{-(1 / 4) \alpha^{2} x}-\frac{e^{-\alpha^{2} x / 4(1+y)}}{\sqrt{1+y}}\right) \frac{d y}{y} d x \\
& +\frac{\sqrt{\pi}}{4} \int_{1}^{\infty} x^{-s+1 / 2} e^{-(1 / 4) \alpha^{2} x} d x \int_{1}^{\infty} e^{-y} \frac{d y}{y} \\
& -\frac{\sqrt{\pi}}{4} \int_{1}^{\infty} x^{-s+1 / 2} \int_{1}^{\infty} \frac{e^{-\alpha^{2} x / 4(1+y)}}{y \sqrt{1+y}} d y d x .
\end{aligned}
$$

We see that the first three integrals are entire and that the last integral devided by $\Gamma(s)$ is entire except a simple pole at $s=1$. By the calculus of residues, we see that $I_{14}(s) / \Gamma(s)$ is entire, and hence $I_{9} / \Gamma(s)$ is entire.

We are left to treat $I_{4}$.

$$
\begin{aligned}
I_{4}= & \frac{1}{6} \int_{0}^{1} x^{s-1}\left(\int_{0}^{1}+\int_{1}^{\infty}\right) r^{2} \sin (\alpha r) e^{-r^{2} x} d r d x \\
& -\frac{1}{3} \int_{0}^{1} x^{s-1} \int_{0}^{\infty} \frac{r^{2} \sin (\alpha r)}{e^{2 \pi r}+1} e^{-r^{2} x} d r d x \\
= & \left(I_{16}+I_{17}\right)+I_{18}, \quad \text { say } .
\end{aligned}
$$

$I_{16} / \Gamma(s)$ and $I_{18} / \Gamma(s)$ are entire. To treat $I_{17}$ we put

$$
\begin{aligned}
& \eta(x)=\int_{1}^{\infty} r^{2} \sin (\alpha r) e^{-r^{2} x} d r, \\
& \eta_{1}(x)=\int_{1}^{\infty} r^{-2} \sin (\alpha r) e^{-r^{2} x} d r
\end{aligned}
$$

and

$$
\eta_{2}(x)=\int_{1}^{\infty} r^{-3} \cos (\alpha r) e^{-r^{2} x} d r
$$

Then for $x>0$,

$$
\begin{aligned}
\eta(x)= & \frac{\sin \alpha}{2} \frac{e^{-x}}{x}+\frac{\alpha \cos \alpha}{4} \frac{e^{-x}}{x^{2}}+\frac{e^{-x}}{4 x^{2}} \sin \alpha \\
& -\frac{1}{4 x^{2}} \eta_{1}(x)+\frac{e^{-x}}{8 x^{3}} \alpha \cos \alpha-\frac{\eta_{2}(x)}{4 x^{3}} \alpha \\
& -\frac{\alpha^{2}}{8} \sin \alpha \frac{e^{-x}}{x^{3}}-\frac{\alpha^{3} \cos \alpha}{16} \frac{e^{-x}}{x^{4}} \\
& +\frac{\alpha^{4}}{16} \frac{\eta_{1}(x)}{x^{4}}+\frac{\alpha^{3}}{8} \frac{\eta_{2}(x)}{x^{4}}
\end{aligned}
$$

We put $F_{1}(s-3)=\int_{0}^{1} x^{s-3} \eta_{1}(x) d x$ and 


$$
F_{2}(s-4)=\int_{0}^{1} x^{s-4} \eta_{2}(x) d x
$$

Then $F_{1}(s-3)$ is regular in $\operatorname{Re} s>2$ and $F_{2}(s-4)$ is regular in $\operatorname{Re} s>3$. We remark that in $\operatorname{Re} s>3$,

$$
\begin{aligned}
F_{2}(s-4)= & \frac{\eta_{2}(1)}{s-2}+\frac{\cos \alpha}{2}-\frac{1}{s-2}(\Gamma(s-3)-E(s-3)) \\
& -\frac{\alpha}{2(s-2)} F_{1}(s-4)
\end{aligned}
$$

and

$$
\begin{aligned}
F_{1}(s-3)= & \frac{\eta_{1}(1)}{s-2}+\frac{\sin \alpha}{2(s-2)}(\Gamma(s-2)-E(s-2)) \\
& +\frac{\alpha \cos \alpha}{4(s-2)}(\Gamma(s-3)-E(s-3))-\frac{1}{2(s-2)} F_{1}(s-3) \\
& -\frac{\alpha^{2}}{4(s-2)} F_{1}(s-4)-\frac{\alpha}{2(s-2)} F_{2}(s-4)
\end{aligned}
$$

where $E(s-2) \equiv \int_{1}^{\infty} x^{s-3} e^{-x} d x$ is entire.

Hence in $\operatorname{Re} s>3$,

$$
\begin{aligned}
& \frac{\alpha^{2}}{4} \frac{s-3}{s-2} F_{1}(s-4)=-\left(s-\frac{3}{2}\right) F_{1}(s-3)+\eta_{1}(1) \\
& \quad+\frac{\sin \alpha}{2}(\Gamma(s-2)-E(s-2))+\frac{\alpha \cos \alpha}{4}(\Gamma(s-3)-E(s-3)) \\
& \quad-\frac{\alpha}{2(s-2)} \eta_{2}(1)-\frac{\alpha \cos \alpha}{4(s-2)}(\Gamma(s-3)-E(s-3)) .
\end{aligned}
$$

From these relations we see that $F_{1}(s)$ and $F_{2}(s)$ can be continued analytically to the whole complex plane except simple poles at $s=-1$, $-2, \cdots$.

Now

$$
\begin{aligned}
6 I_{17}= & \int_{0}^{1} x^{s-1} \eta(x) d x \\
= & \frac{\sin \alpha}{2}(\Gamma(s-1)-E(s-1))+\frac{\alpha \cos \alpha}{4}(\Gamma(s-2)-E(s-2)) \\
& \quad+\frac{\sin \alpha}{4}(\Gamma(s-2)-E(s-2))-\frac{1}{4} F_{1}(s-3)
\end{aligned}
$$




$$
\begin{aligned}
& +\frac{\alpha \cos \alpha}{8}(\Gamma(s-3)-E(s-3))-\frac{1}{4} \alpha F_{2}(s-4) \\
& -\frac{\alpha^{2} \sin \alpha}{8}(\Gamma(s-3)-E(s-3))-\frac{\alpha^{3} \cos \alpha}{16}(\Gamma(s-4)-E(s-4)) \\
& +\frac{\alpha^{4}}{16} F_{1}(s-5)+F_{2}(s-5)
\end{aligned}
$$

Hence we see that $I_{17} / \Gamma(s)$ is entire except, at most, simple poles at $s=$ 4, 3, 2 and 1 . However as we see immediately by calculating the residues that $I_{17} / \Gamma(s)$ is entire.

Thus we have proved that $Z_{\alpha}(2 s-1)$ is entire.

\section{REFERENCES}

[1] Delsarte, L., Formules de Poisson avec reste, J. Analyse Math., 17 (1966), 419 431.

[2] Fujii, A., On the uniformity of the distribution of the zeros of the Riemann zeta function (II), Comment. Math. Univ. St. Paul, 31 (1982), 99-113.

[ 3 ] — The zeros of the Riemann zeta function and Gibbs's phenomenon, Comment. Math. Univ. St. Paul, 32 (1983), 229-248.

[4] Guinand, A. P., A summation formula in the theory of prime numbers, Proc. London Math. Soc. Ser. 2, 50 (1945), 107-119.

[5] Hejhal, D., The Selberg trace formula for $P S L(2, R)$, Lecture notes in Math. Vol. 548, Springer, 1976.

[6 ] - The Selberg trace formula and the Riemann zeta function Duke Math. J., 43 (1976), 441-482.

[ 7 ] Kubota, T., Elementary theory of Eisenstein series, Halsted Press, New York, 1973.

[8] Minakshisundaram, S. and Pleijel, A., Some properties of the eigenfunction of the Laplace operator on Riemannian manifolds, Canad. J. Math., 1 (1949), 242-256.

[9] Selberg, A., Harmonic analysis and discontinuous group in weakly symmetric Riemannian spaces with applications to Dirichlet series, J. Indian Math. Soc., 20 (1956), 177-189.

[10] Venkov, A. B., Selberg's trace formula for the Hecke operator generated by an involution and the eigenvalues of the Laplace-Beltrami operator on the fundamental domain of the modular group $P S L(2, Z)$, Math. USSR-Izv., 12 (1978), $448-462$.

[11] _- Remainder term in the Weyl-Selberg asymptotic formula, J. Soviet Math., 17 (1981), 2083-2097.

Department of Mathematics

Rikkyo Univ.

Tokyo, Japan 